\title{
Reconciliation of science requirements with technological possibilities: final discussion
}

\author{
Compiled by M. Dennefeld ${ }^{1}$, B. Leibundgut ${ }^{2}$ \& P. A. Whitelock ${ }^{3,4,5}$ \\ ${ }^{1}$ Institut d'Astrophysique, Paris, France \\ email: dennefel@iap.fr \\ ${ }^{2}$ European Southern Observatory, Garching bei München, Germany \\ email: bleibund@eso.org \\ ${ }^{3}$ South African Astronomical Observatory, Cape Town, South Africa \\ ${ }^{4}$ NASSP, Department of Mathematics and Applied Mathematics, University of Cape Town, \\ South Africa \\ ${ }^{5}$ Department of Astronomy, University of Cape Town, South Africa \\ email: paw@saao.ac.za
}

\begin{abstract}
This paper presents the final discussion of the meeting, following introductory remarks by A. Ardeberg, B. Ellerbroek and C. Cunningham.
\end{abstract}

Keywords. Instrumentation: adaptive optics, spectrographs; Telescopes

\section{Introduction}

Following the summary talks of the four parallel sessions (see papers by Hook, Mould, Theuns and McCaughrean in these proceedings), in which the chairs presented some specific requirements, the SOC initially intended a panel discussion to "confront" the wishes from the scientists with the possibilities offered from the technical side. To help focus the discussion a number of questions had been assembled and made available to all participants on the previous day; these fell into two categories: technical and organisational. As so many experts were present at the meeting, rather than select only a few of them to participate in the panel, the SOC decided to have a fully open discussion introduced by some luminary remarks on key-issues. These are presented below, before the transcription of the discussion itself.

\section{Suggested questions}

For each of the parallel sessions, what were the:

- Priorities

- Major technical requirements on:
- spatial resolution
- contrast ratio
$\circ$ wavelength range
o field of view
- atmospheric turbulence compensation
$\circ$ effect of wind forces
- instrumentation
- telescope size 


\section{Organisational questions:}

- Number of ELTs: one doing everything, or several specialized?

- Time scales: small and early, or big and late?

- What are the required synergies with other projects, e.g. JWST, ALMA, SKA, etc.?

- All sky coverage, or cheaper limited coverage, like SALT?

- Operations: few, large-scale programs, or many "smaller" ones?

- What will be the role of other, "smaller", telescopes?

- How can we best achieve the essential iteration between the science cases, the telescope design and the instrumentation?

\section{Luminary remarks}

\subsection{Introductory notes from A. Ardeberg}

\section{Many factors are important}

There are a number of "specifications" of high concern, some of which are in mutual conflict. These specifications include the spatial resolution, the contrast ratio, the wavelength range, the field of view, the compensation of atmospheric turbulence and correction for the effects of wind forces. A serious concern is the often somewhat neglected but highly important interfacing of instrumentation and telescope.

\section{Spatial resolution}

Diffraction-limited operation is regularly assumed. However, such operation stresses a number of difficult arrangements. Important issues concern the optical surfaces, the alignment of the opto-mechanical system, the related metrology as well as the mechanical structures and mirror supports. Furthermore, it assumes highly demanding compensation of several effects. Such effects are produced by gravity, temperature, wind forces, atmospheric turbulence, including speckles, satellite images (segmented primary mirror) and stray light caused by mirror surfaces not ideally clean.

Every one of these effects implies a serious problem. Together, they define a very significant challenge. All in all, we are left with very little room for imperfections. Almost everything has to work in a close to perfect manner.

\section{Strehl ratio}

Very ambitious science-case programmes put high demands on the Strehl ratio. Quite often, there seems to be a gap between these demands and what is feasible. For ELT first light, we can, realistically, state that a Strehl ratio of 0.2 is not trivial. Strehl ratios of 0.3-0.4 are already considerable challenges, while a corresponding ratio of 0.5 is very challenging indeed. At least for first-light operation, Strehl ratios of 0.6 and larger seem somewhat optimistic.

Fortunately, a telescope with at Strehl ratio of 0.3 can deliver very impressive data. Higher Strehl ratios, as well as comparable values for shorter wavelengths, will come but maybe not be with first light. Limitations will be imposed by optical surfaces, actuator precision, actuator density versus size, wavefront measurements and the stability of corrections.

\section{Halo hole}

We would like to decrease the level of the halo. Equally important, we want to widen the halo hole. For this arrangement, the fitting error is dominant. 
Planets, not least the Earth-like ones, have to be observed in the halo hole. Thus, the halo hole has to be as clean as possible. However, it is partly filled by the effects of the aliasing error, caused by discrete wavefront sampling, and the servo-lag error, due to delay in the surface fitting of the deformable mirror(s). It is also filled by the detectionnoise error coming from measurement noise of the wavefront data. Further, filling effects are caused by the anisoplanatic error, resulting from measurements and corrections of wavefronts made at different angles, and by the chromatic error, correspondingly due to measurements and corrections of wavefronts made at different wavelengths.

\section{Contrast ratio}

Science demands on the contrast ratio are often very high, especially in connection with Earth-like planets. Requirements are, in these cases, of the order of $10^{9}$ to $10^{1} 0$ and even larger, depending on several factors. Again, almost everything has to work ideally, or no planet will be observed. The contrast ratio required for studies of Earth-like planets is an exceptionally hard challenge.

\section{Wavelength range}

The science-case requirements on the wavelength range are from $400 \mathrm{~nm}$ to $25 \mu \mathrm{m}$. For wavelengths shorter than $1-2 \mu \mathrm{m}$, adaptive optics operation will be a major challenge, especially initially. On the other hand, wavelengths larger than around $5 \mu \mathrm{m}$ will suffer from difficulties with the atmosphere and with the telescope.

\section{Field of view}

Many science programmes need a large field of view. However, the isoplanatic angle is normally less than 5 arcsec in visual light. Thus, direct adaptive-optics operation will be applicable to limited fields only. Single-conjugate adaptive optics will cover fields up to one arcmin. One natural guide star may suffice. However, if laser guide stars are used, the cone effect implies that several such guide stars are required, plus at least one natural guide star. Multi-conjugate adaptive optics can cover fields up to two arcmin, but need at least two natural guide stars and several laser guide stars. With ground-layer adaptive optics, larger fields can be covered, up to several arcmin.

With laser guide stars, the effect of perspective elongation must be taken into account. If the elongation is larger that the isoplanatic patch, we are in serious trouble. This effect needs further consideration.

\section{Wind forces}

Wind forces on the telescope, and not least on the primary mirror, are a serious problem. For compensation, we have to apply a relatively large bandwidth. However, the amplitudes caused by wind forces are quite large and increase with the size of the aperture. A major part is due to effects of tilt, but a very large suppression factor is needed, of the order of $10^{5}$. The challenge concerning wavefront sensors and edge sensors is impressive.

\section{Instrumentation and telescope}

High-quality observational data require not only an excellent telescope but equally excellent instrumentation. In addition, and very important, they demand a high-quality interface between telescope and instrumentation. Also, an important requirement concerns gravity-invariant and spacious instrumentation platforms. A natural way to achieve an optimal design is to co-tailor telescope and instrumentation. Even better, and of course quite natural, is to maintain a constant communication between the teams working on the science case, the telescope and the instrumentation. In this sense, general practice 
leaves room for improvement. An ELT operation corresponding to the high ambitions and requirements of science needs these improvements.

\subsection{Remarks by B. Ellerbroek}

I would first like to thank, in the name of the audience, the organisers for a well thoughtof scientific program.

My remarks will be restricted to Multi-Objects Adaptive Optics (MOAO).

Astronomers should be cautioned against expecting too much from MOAO in the first generation of ELT instrumentation, since it:

(i) relies upon significant advances in a relatively immature component technology (MEMS DMs) in terms of stroke, order of correction, and linearity;

(ii) relies upon new and largely untested concepts for open-loop wavefront correction, with no direct optical feedback of the corrections provided by the MEMS

(iii) is not receiving significant attention in the current generation (and next generation) of $\mathrm{AO}$ instrumentation under development for large telescopes.

It would be more prudent and realistic to develop road-maps which envision MOAO as $2^{\text {nd }}$ generation capabilities following prior work on the "building block" technologies of MEMS and IFUs.

\subsection{Comments by C. Cunningham}

I would like to repeat a couple of points from my presentation.

First, I think it is very important that we take a systems approach to satisfying the scientific aims developed at this symposium. The complete telescope, adaptive optics, instrumentation and data processing package needs to be optimised in order to get the best results from what will be very expensive facilities.

Secondly, it would be very beneficial for the entire world astronomical community if we could all work together to optimise what could be several ELTs and avoid duplication of capability, particularly where specific sites or telescope designs offer major advantages. If one telescope could be optimised for high spatial resolution, narrow field astronomy such as exoplanet studies, another for wide-field high-redshift galactic work, and a further for thermal IR and maybe sub-mm science we could get more capability for less total expenditure. Of course, we would also need to collaborate over telescope access as well.

During discussions, several people agreed with the latter point, but noted the difficulty of coordinating agencies and funding to achieve this end. One point made was that it would make sense to maintain communications between the telescope teams to help come to informal agreements as to what would form complementary sets of first light instruments. It was also agreed that coordination for $\mathrm{R} \& \mathrm{D}$ is very desirable.

\section{Discussion}

KäUfL: Two remarks: first, the wavelength range to be covered is so large (from the UV cutoff to the thermal IR) that probably more than one ELT is needed if one wants to optimize them.

Second, the trade-offs between MCAO (presumably first) and MOAO (coming later) need to be explored. 
QUIRREnBACH: Has the telescope to be designed directly for MOAO, or is there space for that to come later?

SHAVER: How do "scientific breakpoints" influence telescope size? Are there any and are they important? Is the case of the resolved population in Virgo galaxies the only one left for the $100 \mathrm{~m}$ telescope?

Colless: The Field of View might be one. Should one think about smaller telescopes with moderate AO (and large FoV) and a larger telescope with strong AO to study the details of individual objects? How can the trade off between field of view and good AO correction be solved?

DENNEFELD: Should telescopes really be specialised for certain science applications? Or can this be done at the level of the instruments?

Ellerbroek: Maybe one should think about one telescope at a high site (for IR or sub-mm use) and another one at a good seeing site at a lower altitude? Another issue is the high segmentation and its difficulty for AO.

Colless: Can GMT or TMT designs still be changed? e.g. is the diameter fixed already now?

MCCARThy: For the GMT this is already fixed. After all the first mirror is already cast!

MOULD: In the case of the TMT this is still up for a later review.

ZINNECKER: One should consider the size to reach the same resolution as ALMA. This is important for fields like star formation. At $2 \mu \mathrm{m}$, this would imply a diameter of $40 \mathrm{~m}$.

MCCARThY: At $1 \mu \mathrm{m}$, this is OK with the GMT.

QuirRenBACH: It is hard to deal with data with Strehl ratios of 0.2 or 0.3 , for instance, and the same applies in other areas. One needs to develop user-friendly tools, particularly for AO data analysis. So far, it appears that nothing has been planned. In the case of ALMA this aspect is covered by the project. What about the ELTs? Could there be a possibility of a collaboration between the individual projects?

Monnet: What about IFU data in the future? Will there anything be done in this area, e.g. reproduction of the PSF from IFU data themselves? Full data exploitation is absolutely required.

LONGAIR: It seems unavoidable that ambitious and complex projects like the European OWL will have to be simplified to meet budgetary constraints. One needs to think again about the instruments design, to make them simpler and more realistic.

Cunningham: The OWL instrument studies were not meant to be first light instruments, but rather to push the design. The instruments seeing first light will be much simpler, but must have ability to upgrade.

MOULD: The shortage of money will drive towards specialised projects rather than a single one with a very wide range of abilities. How will this happen? It seems important 
to have a "coordinated system" between the projects. Good communication between them is essential. However, this will probably sort itself out "automatically."

Cullum: The calibration of instruments is important. Atmospheric dispersion, for instance, will not be easy to correct, but is often taken for granted. Also, the complicated PSFs will have to be handled. What about the diffraction spikes? More work is needed on those aspects, particularly for GLAO.

Monnet: In the case of GLAO, this is not a problem because of the large pixels.

ELLERBROEK: The photometric accuracy and constancy with GLAO needs to be investigated.

Crampton: For Gemini this has not been a problem. It also strongly depends on the science application.

Dravins: One should keep the science cases open. The future science will probably be different from what has been discussed so far.

DEnNEFELD: How will the instruments be prioritised in GMT and TMT?

MCCARTHY: With the first generation instruments, the parameter space should be covered so that all conditions can be used. After this, further discussion will take place.

Monnet: The discussion for OWL has started, but the choice of instruments depends on the site! Point designs will be evaluated next.

KäUfL: What can be done with partially filled apertures? This should also influence the choice of the first instruments.

DENNEFELD: What about the synergy with space missions? Does an ELT have to be contemporaneous with a space mission?

MoulD: It would be good to have an iterative process for the synergy.

MCCARThy: This is very important and an important goal for the GMT! The example of HST and Keck (but not VLT and Gemini) is key here. For JWST, we cannot compete in sensitivity, but will complement it in using other wavelengths.

DENNEFELD: We are talking about studies of distant galaxies, where we measure (in the visible) redshifted UV light. Yet we know very little about UV properties of the local universe for comparison, and there is currently no UV facility available for spectroscopy (unless the HST is repaired), and nothing is planned in the next two decades either. Here we need action in the space domain, rather than on the ground!

BAADE: The data products from the ELTs must be comparable between the different projects. Also, the current photometric standards are all based on data from very small telescopes. The preparation for future calibrations needs to start now. Should one think about coordinating the optical filter definitions? Should the site surveys include extinction measurements with the site monitors and a comparison between different sites?

Gilmore: It is dangerous to tie ELTs too strongly to JWST. The ELTs will have life 


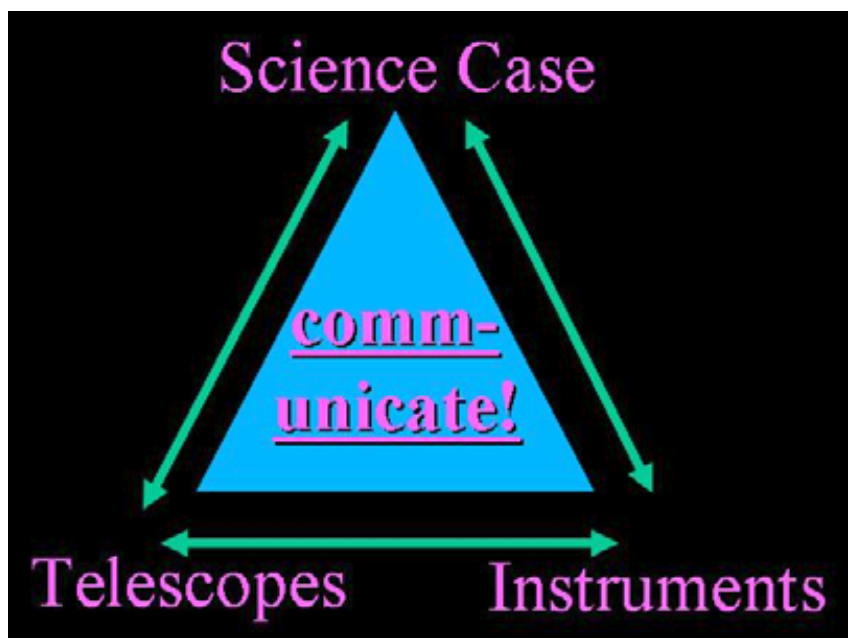

Figure 1. The required communication triangle.

times of 30 years, space missions are much shorter. One should also consider synergies with Planck or other ground/space projects. A recommendation has recently been adopted by the ESO council: "Europe must not be late".

ZiJLSTRA: Reduction software and pipelines are important issues. They must be available at an early phase, for the astronomers to make use of the data.

DENNEFELD: Our available time is coming to an end. But such discussions need to continue in the future. What are the solutions? Should there be regularly international meetings of this type? Should one meet along other, national, meetings? Could one have science meetings in parallel with other technical meetings?

MCCARThy: Next opportunity could be the SPIE meeting in Orlando.

Hook: We need support for astronomers to attend the expensive SPIE meetings.

WALton: There will be a meeting on ELTs during the IAU General Assembly in Prague, in August 2006.

CRAmpton: I am happy to have as much help as possible from anyone! Everybody is invited to attend TMT meetings if they wish so.

DENNEFELD: Thanks to all of you for very useful input. Such discussions should help to better define the projects, and we hope they will go on in the future, opening ways for further collaborations, and development of some major telescopes as soon as possible. Thanks to our hosts for their nice hospitality, thanks to all of you for coming, and have a nice and safe trip back home.

\section{Conclusions}

Keep the loop closed between scientific requirements, telescope designs, and instrumental developments (Fig.1)! 


\section{Acknowledgements}

It is a pleasure to thank the students from South Africa: Abiy Tekola, Paul Kotze, Patrice Okuma, Nebiha Shafi, Ros Skelton, Bonita de Swardt, Angel Torres Rodriguez, under coordination from Lisa Crause, for distributing and collecting the question sheets during the whole meeting. We hope to see them again soon in our professional circles. 